\title{
IDR/UPM Facilities for Liquid Bridge Experimentation on Earth under Microgravity Conditions
}

\section{Introduction}

A liquid bridge consists of an isothermal drop of liquid held by surface tension forces between two parallel, solid disks as shown in Fig. 1. Disregarding electric and magnetic fields effects, the equilibrium interface shape and hydrostatic stability limits of such a fluid configuration are determined by the slenderness, $A=L /\left(2 R_{0}\right)$, where $L$ is the distance between the supporting disks and $R_{0}=\left(R_{1}+R_{2}\right) / 2$ is the mean radius; the ratio of the radius of the smaller disk, $R_{1}$, to the radius of the larger one, $R_{2}$, that is $K=R_{1} / R_{2}$, or the equivalent parameter $h=$ $(1-K) /(1+K)=\left(R_{2}-R_{1}\right) /\left(R_{2}+R_{1}\right)$; the dimensionless eccentricity, $e-E / R_{0}, 2 E$ being the distance between the disks axes; the dimensionless volume, defined as the ratio of the actual volume $V_{f}$ to the volume of a cylinder of the same length $L$ and diameter $2 R_{0}: V=V_{f} /\left(\pi R_{0}^{2} L\right)$; the axial Bond number,

component of the acceleration acting on the liquid, and $\sigma$ is the surface tension; the lateral Bond number, $B_{l}=\Delta \rho g_{l} R_{0}^{2} / \sigma$, where $g_{\text {? }}$ stands for the lateral component of the acceleration acting on the liquid, which forms an angle $\beta$, with respect to the plane defined by the axes of the disks, and the Weber number (assuming that the liquid bridge is rotating as a solid body with angular velocity $\omega), W=\Delta \rho \omega^{2} R_{0}^{3} / \sigma$.
$B_{a}=\Delta \rho_{a} R_{i}^{2} / \sigma$, where $\Delta \rho$ is difference between the density of the liquid and the density of the surrounding medium, $g_{a}$ is the axial

The beginning of the studies on liquid bridge behaviour at the Universidad Politécnica de Madrid dates back to 1974, when Prof. Da Riva (\$1991) answered an ESA Call for Ideas for Spacelab experiments, suggesting a detailed analysis of the stability of liquid bridges $[1,2]$. Although the effort was initially concentrated on the preparation of this experiment, the existence of other flight opportunities gave way to a much broader research program, so that together with theoretical studies a large effort was devoted to ground simulation of microgravity conditions. 
Concerning liquid bridges theoretical approach, a great effort has been done world wide in the last decades to determine equilibrium shapes and stability limits for a wide range of liquid bridge configurations (a review of the literature dealing with these effects can be found in $[3,4]$ ). Besides, numerous papers on liquid bridge dynamics have been also published. The nonlinear dynamics and breakage of liquid bridges was first studied some twenty years ago, using inviscid, one-dimensional models $[5,6]$. An historical review of the most representative publications concerning the breaking of liquid bridges can be found in $[7,8]$,

Real experiments on microgravity demand cancelling gravity with inertia forces (taking into account the definition of Bond number that means that the resulting acceleration acting on the liquid column vanishes, $g \rightarrow 0$ ), thus recourse is had to free fall towers, aircraft parabolic flights, sounding rockets and orbiting platforms, depending on the time required. All these recourses have been used at IDR/UPM, with different experiments performed in Spacelab-1 Mission, Spacelab-D1 Mission, SpacelabD2 Mission, TEXUS sounding rockets, and others.

Concerning Earth experimentation, most research teams have developed some kind of Plateau tank for their experimental work. A Plateau tank is just a reservoir filled with liquid inside which another liquid, immiscible with the former and with the same density $(\rho \Delta \rightarrow 0)$, can be studied as in weightlessness (in

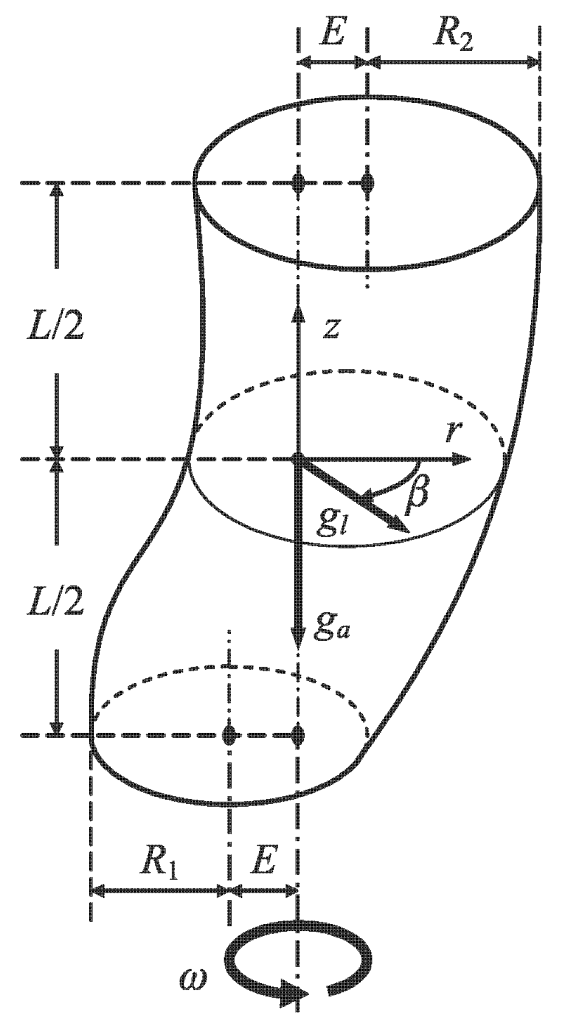

Fig. 1: Sketch of the liquid bridge. some respects). Of course this method does not simulate completely orbital conditions because of the presence of the outer liquid, which affects the dynamics of the fluid column [9], but seems to be very appropriate to hydrostatic analyses. Other methods of minimizing gravity effects can be to reduce the size of the experiment to a very small scale $\left(R_{0} \rightarrow 0\right)$, say a millimetre or less.

\section{Platean Tank Facility}

The primary goal of this Plateau Tank Facility (PTF) was to serve as a ground support experimental facility for the Spanish experiment 1-ES-331, performed with the Fluid Physics Module (FPM) on Spacelab 1. The main design philosophy was to get a Plateau tank similar to the test chamber of the FPM [10],

Table 1: Some published papers dealing with experimental studies on liquid bridges. Numbers in the table indicate, grouped by used facility, the paper number according to the list of references. PTF: Platean Tank Facility, TOPT: Tele-Operated Plateau Tank, TORF: Tele-Operated Rotating Facility, MLBF: Millimetric Liquid Bridge Facility. The first column indicates the disks configuration: equal ( $h$ $=0$ ) or unequal in diameter disks $(h \neq 0)$; coaxial $(e=0)$ or noncoaxial disks $(e \neq 0)$.

\begin{tabular}{lcccc}
\hline Configuration & PTF & TOPT & TORF & MLBF \\
\hline$h=0, e=0$ & $6,9,11,12,14$ & 19 & 21 & $8,23,25,26,28,29$ \\
\hline$h \neq 0, e=0$ & 13 & 18 & & 4,22 \\
\hline$h=0, e \neq 0$ & & & & 24 \\
\hline$h \neq 0, e \neq 0$ & & & & 27 \\
\hline
\end{tabular}

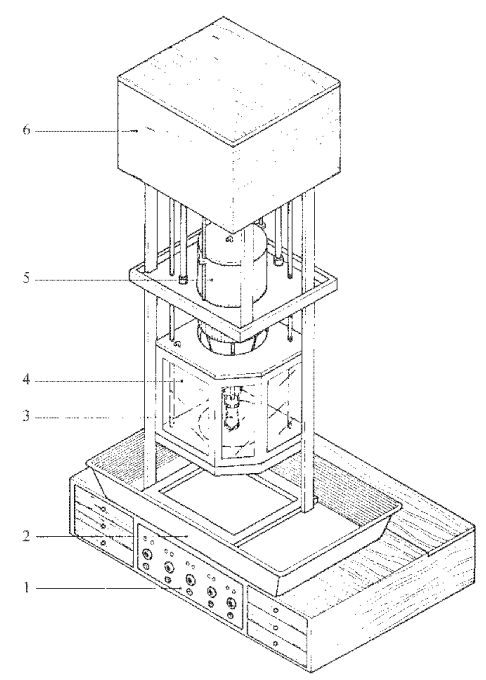

Fig. 2: Sketch of the PTF: 1) Control panel. 2) Safety pan. 3) Liquid bridge. 4) Plateau tank. 5) Upper disk cylinder. 6$)$ Motor box. 
but free from other subsystems that render this flight module extremely complex. Hence, at its more crude description, the PTF is but a glass box screwed to a motor box through broken shafts.

The PTF comprises a glass test chamber held in a tubular structure (Fig. 2). Inside the tank there are the two parallel discs to form the zone and transmit all the mechanical perturbations envisaged. The upper disk has two movements, rotation (in both senses) and axial displacement, whereas the lower disk has three movements, rotation (in both senses) and axial vibration and lateral displacement [10].

The major experiments performed on this Plateau Tank Facility were devoted to the study of the breakage of initially cylindrical zones near the stability limit as well as the analysis of liquid bridge oscillations. These studies are summarized in Table 1. Most of these papers are related to liquid bridges between coaxial, equal in diameter circular disks, the performed experiments being concerned with the dynamics of liquid bridges like the breaking [6], the liquid column oscillations, either axial [9] or lateral [12], or related aspects of the injection of liquid to form the bridge [14]. This equipment has been also used for hydrostatic studies, both for configurations between disks of equal diameter [11] and of unequal one [13].

\section{Tele-Operated Plateau Tank Facility}

From the lessons learned with the work performed with the previous facility (PTF) an Tele-Operated Plateau Tank (TOPT)

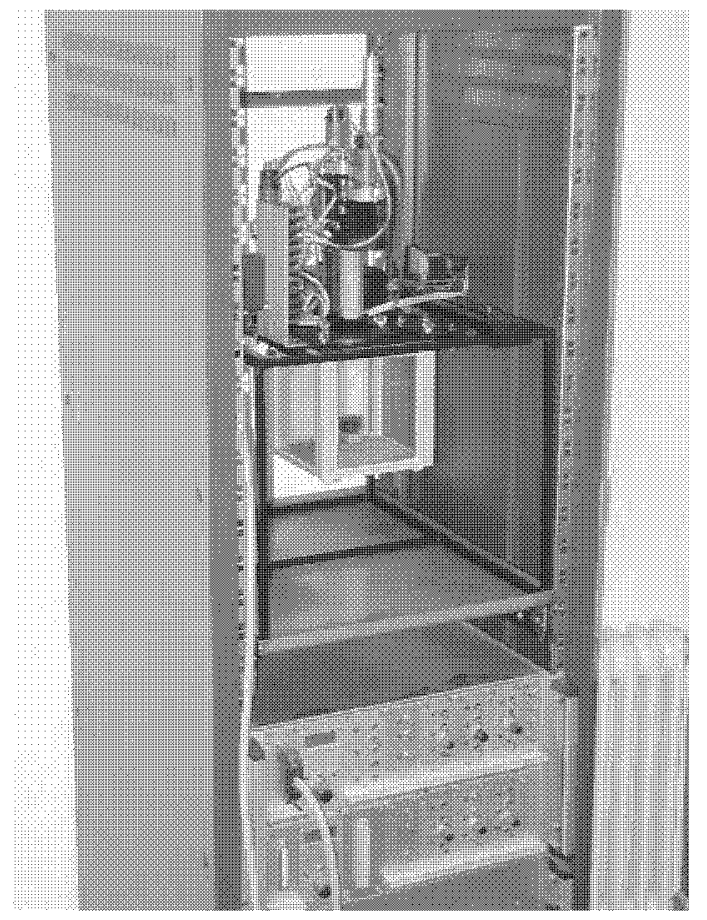

Fig. 3: Photograph of the Tele-Operated Plateau Tank Facility showing the liquid cell at the top side, where liquids and notors are located, and the electronics bot (where electronic boards for driving the motors are located). facility was developed [15-17]. This facility can be remotely controlled to bring the possibility of using it in telescience operations. A view of the TOPT is shown in Fig. 3. It consists of three main blocks: the liquid cell, where liquids and motors are located, the electronics box (where electronic boards for driving the motors are located), and the controller, a microcomputer with data acquisition and image processing capabilities (not shown in the photograph). The liquid cell can be driven either directly by means of a control panel in the electronics box or remotely via the attached computer.

Two units of the TOPT were manufactured, one of them was supplied to ESA/ESTEC for on-Earth astronauts training, and the second was used at IDR/UPM for different experimental studies, some of them indicated in Table 1, one concerning the minimum volume stability limits of liquid bridges between unequal disks [18], and the second on the influence of the viscosity on the oscillation of axisymmetric liquid bridges [19].

\section{Tele-Operated Rotating Facility}

This experimental facility consists of a liquid bridge cell mounted on a horizontal, rotating platform [20]. The rotating platform is a metallic beam $1.5 \mathrm{~m}$ in radius, mounted on a support structure where the electric motor used to rotate the beam as well as the control electronics are located (Fig. 4).

The rotating platform supports the test box as well as the data and commands transmission unit, which is located at the platform rotation axis. The test box consists of two rectangular

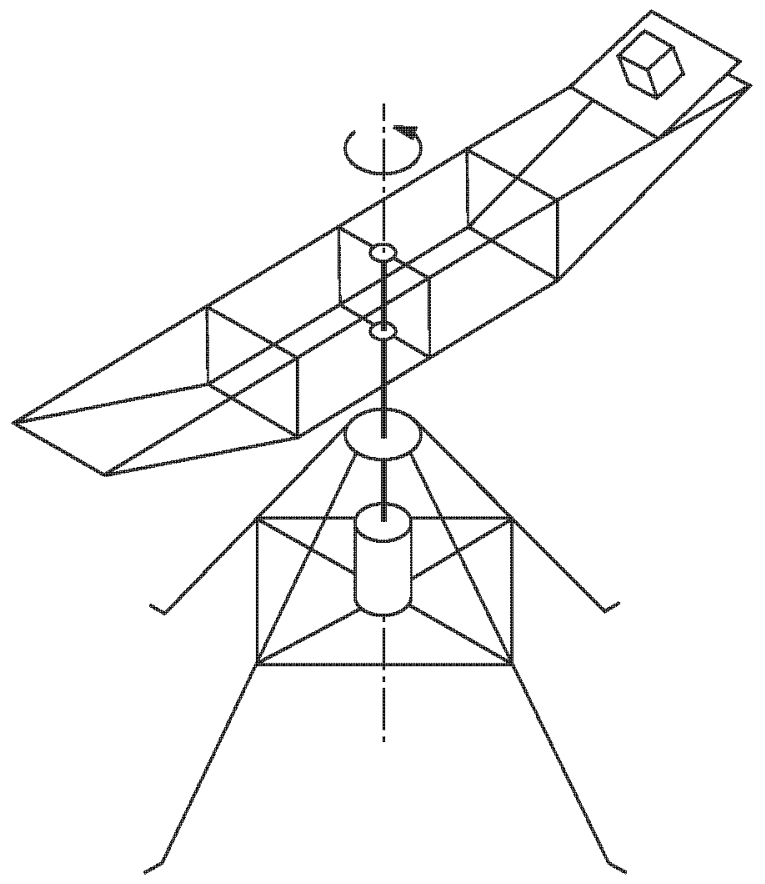

Fig. 4: Sketch of the Tele-Operated Rotating Facility (TORF): a liqwid bridge cell mounted on a horizontal, rotating platform which in whn is motnied on a stuport stucture. 
plates with a hinge mounted on a common side. One of the plates is attached to the rotating platform whereas the second one can be rotated at any prescribed angle with respect to the former. Upper plate supports the liquid bridge cell, a tight Plateau chamber connected to a calibrated syringe. To monitor the system performance a $\mathrm{CCD}$ video camera is used, together with a TV sender and a TV receiver.

This equipment has been used to experimentally analyze the effect of acceleration gradients on the stability of long liquid bridges [21]. The facility has been modified to accommodate different payloads and now is being used for aerodynamic studies on the effect of moving bodies on static ones.

\section{Milimetric Liquid Bridge Facility}

The so called Millimetric Liquid Bridge Facility (MLBF) is rather a set of deviees that can be arranged in different ways to obtain customized assemblies than an integrated equipment (Fig. 5). This experimental facility consists of the following main elements: liquid bridge cell, microscope, high speed video camera, normal rate CCD cameras, illumination systems, image recording system and associated software.

The liquid bridge cell consists of a three-axes table, the displacement along each one of the different axes being controlled through micrometric serews. The bottom disk is mounted on the platform ( $x-y$ plane) of the three-axes table, and it can be displaced both along the $x$-axis and the $y$-axis. The upper disk is mounted on the z-guide of the table, so that its displacement can only be along the z-axis. Liquid bridge supports (disks) are made of calibrated stainless-steel tube or brass tube, typical disks diameters used in experiments range from $0.8 \mathrm{~mm}$ to 1.5 mm.

When moderate thermal gradients must be imposed to the liquid column, each one of the supporting tubes is mounted on small blocks of plastic material which in turn are hollow. Therefore, by circulating either cold or hot liquid through the

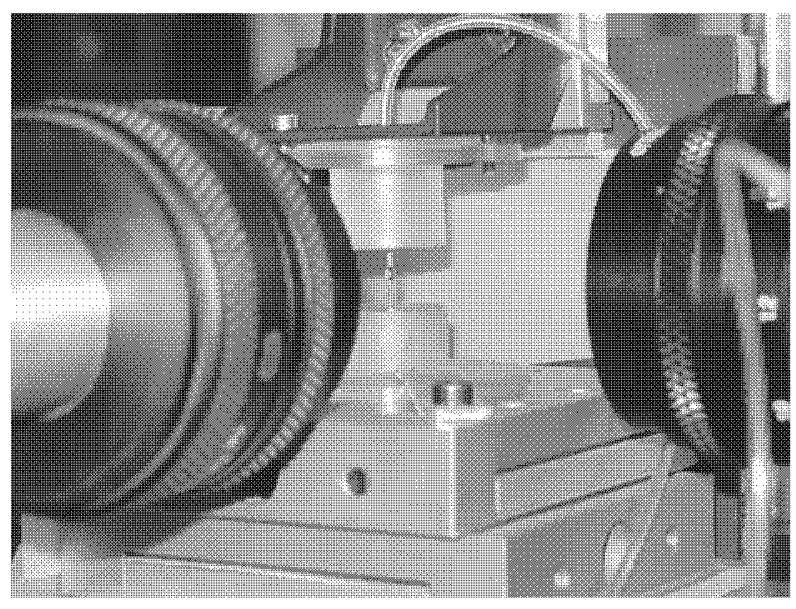

Fig. 5: View of a typical configuration of the Millimetric Liquid Bridge Facility (MLBF) hollow plastic blocks the supporting tubes are cooled (or heated). Another possibility for the hot support is to heat it by using an electric heater thermally linked to the supporting tube [28].

MLBF has been used during more than ten years for a wide spectrum of liquid bridge experiments. As indicated in Table 1 these experiments deal mainly with liquid bridges between coaxial disks of the same diameter, and they have been oriented to the study of the equilibrium shapes and stability of liquid bridges subject to both axial $[4,22,23]$ and lateral accelerations [24-27], to the analysis of the breaking dynamics [4, 22], and to the analysis of thermal problems related to Marangoni flow [28] and the influence of a temperature gradient along the liquid column span on the stability of long liquid bridges [29].

Some representative results obtained by using MLBF are shown in Fig. 6, where the variation with the dimensionless eccentricity, $e$, of the liquid bridge volume at minimum volume stability limit, $V$, of liquid bridges between unequal disks, $h$, under lateral gravity acceleration, $B_{l}$, is presented [27]. In the same plot theoretical predictions according to the asymptotic expression

$V=1+\frac{2}{\pi} \Lambda+2\left(\frac{3}{2}\right)^{4 / 3}\left(\frac{1}{\pi} h\right)^{2 / 3}+\frac{\pi^{2}}{2} B_{l}^{2}+\frac{3}{2 \pi} e^{2}$

are shown.

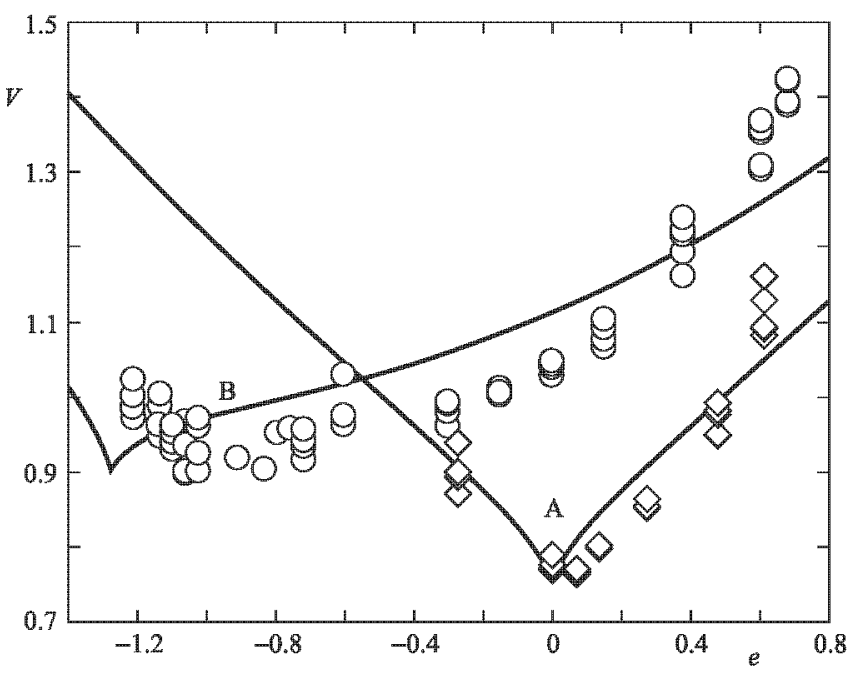

Fig. 6: Dimensionless minimum volume, $V$, versus eccentricity, $e$, of liqud bridges between unequal disks under lateral gravity acceleration. Labels identify the liquid bridge configuration according to the following key: $A) h=0, B l=0.074 ; B) h=0.114, B l=0.059$. Symbols indicate experimental results whereas solid lines correspond to theoretical predictions $[27]$. 


\section{Reterences}

1 Da Riva, 1, Maninez, I.: Floating Zone Stability (Exp. 1.ES-331). ESA SP 142, pp. 67-74 (1979).

2 Da Riva, I., Napolitamo, L.G.: Fluid Physics under Reduced Gravity - an Overvicw. ESA SP 191, pp. 5-12 (1983).

3 Meseguer, J., Perales, J.M., Martinez, I, Bezdenejm,h, N.A., Sanz, A.: Hydrostatic Instabilities in Floating Zone Crystal Growth Process. Curr. Topics Crystal Growth Res., Vol. 5, pp. $27-42$ (1999).

4 Meseguer, J., Espino, J.L., Perales, J.M., Laverón-Simavilla, A.: On the Breaking of Long, Axisymmetric Liquid Bridges between Unequal Supporting Disks at Minimum Volume Stability Limit. Eur. J. Mech. B/Fluids, Vol. 22, pp. 355-368 (2003).

5 Meseguer, J.: The Breaking of Axisymmetric Slender Liquid Bridges, J. Fluid Mech., Vol. 130, pp. 123-151 (1983).

6 Meseguer, J., Sonz, A.: Numerical and Experimental Study of the Dynamics of Axisymmetric Slender Liquid Bridges. J. Fuid Mech., Vol. 153 , pp. 83-101 (1985).

7 Zhang, X., Padget, R.S., Basaran, O.A.: Nonlinear Deformation and Breakup of Stretching Liquid Bridges. J. Fluid Mech., Vol. 329, pp. 207 245 (1996).

8 Espino, J.L., Meseguer, J., Laverón-Sinavilla, A. An Experimental Study of the Breakage of Liquid Bridges at Stability Limit of Minimum Volume. Phys. Fluids, Vol. 14, pp. 3710-3713 (2002).

9 San, A.: The Influence of the Outer Bath in the Dynamics of Axisymmetric Liquid Bridges. J. Fluid Mech., Vol. 156, pp. 101-140 (1985).

10 Martinez, I., Rivas, D.: Plateau Tank Facility for Simulation of Spacelab Experiments. Acta Astronautica, Vol. 9, pp. 339-342 (1982)

11 Sanz, A., Martinez, I. Minimum Volume for a Liquid Bridge between Equal Disks. J. Colloid Interface Sci., Vol. 93, pp. $235-240$ (1983).

12 Sanz, A., López-Diez, J.: Non-Axisymmetric Oscillations of Liquid. Bridges. J. Fluid Mech., Vol. 205, pp. 503-521 (1989).

13 Meseguer, J., Mayo, L.A., Llorente, J.C., Fernández, A.: Experiments with Liquid Bridges In Simulated Microgravity. J. Crystal Growth, Vol. 73 , pp. 609-621 (1985).

14 Sanz, A., Perales, J.M.: Liquid Bridge Formation. Appl. Microgravity Technol, Vol. 2, pp. 133-141 (1989).

15 Meseguer, J., Sanz Andrés, A., Perales, J.M.: Equipo para la experimentación con fluidos en microgravedad simulada (Tele-Operated Plateau Tank Facility). Anales de Química, Vol. 87, pp. 537-540 (1991).

16 Sanz-Andrés, A., Perales, J.M., Rodriguez de Francisco, P, Sanz-Lobera, A.: A Plateau Tank Facility (PTH) for Liquid Bridge Experimentation by Using Buoyancy Technique for Microgravity Simulation. ESA SP-295, pp. 607-611 (1990).

17 Rodriguez de Francisco, P, Samz, A., Perales, J.M., Sant, A.: Design and Manufacturing of an APTF to Test Fluid Behaviour in Microgravity Environment. ESA SP-299, pp. 299-304 (1990).

18 Perales, J.M., Meseguer, J., Martinez, I.; Minimum Volume for Axisymmetric Liquid Bridges Subject to Steady Acceleration. J. Crystal Growth, Vol. 110, pp. 855-861 (1991).

19 Perales, J.M., Meseguer, J.: Theoretical and Experimental Study of the Vibration of Axisymmetric Viscous Liquid Bridges. Phys. Fluids A, Vol. 4. pp. 1110-1130 (1992).

20 Zayas, F, Meseguer, J., Perales, J.M., Sanz, A.: A Teleoperated Facility for Variable Gravity Level Fluid Physics Experimentation. Paper G0.10051, 33rd COSPAR Scientific Assembly, Warsaw, Poland, 16-23 July (2000).

21 Zayas, F, Alexander, J.I.D., Meseguer, J., Ramus, F: On the Stability Limits of Long Non-Axisymmetric Cylindrical Liquid Bridges. Phys. Fluids, Vol. 12, pp. 979-985 (2000).

22 Bezdenejwy h, N.A., Meseguer, J.: Stability Limits of Minimum Volume and Breaking of Axisymmetric Liquid Bridges between Unequal Disks. Microgravity Sci. Technol., Vol. 4, pp. 235-239 (1991).
23 Beudenejny,h, N.A., Meseguer, J., Perales, J.M.: Experimental Analysis of Stability Limits of Capillary Liquid Bridges. Phys. Fluids A, Vol. 4, pp. 677-680(1992).

24 Meseguer, J., Bewdenemykh, N.A., Perales, J.M., Rodrgue de Francisco,

P. Theoretical and Experimental Analysis of Stability Limits of NonAxisymmetric Liquid Bridges. Microgravity Sci. Technol., Vol. 8, pp. 2-9 (1995).

25 Meseguer, J., Bezdenejmhh, N.A., Rodriguez de Francisco, P: On the Use of Liquid Bridges as Accelerometers. Microgravity Sci. Technol., Vol. 9, pp. 62-69(1996).

26 Bezdenejnyh, N.A., Meseguer, J., Perales, J.M.: An Experimental Analysis of the Instability of Non-Axisymmetric Liquid Bridges in a Gravitational Field. Phys. Fluids, Vol. 11, pp. 3181-3185 (1999).

27 Meseguer, J., Espino, J.L., Cuerva, A. Sanz-Andrés, A.: Minimum Volume of Long Liquid Bridges Between Non-Coaxial, Non-Equal Diameter Circular Disks Under Lateral Acceleration. Phys. Fluids, Vol. 17, code 108101, 4 p. (2005).

28 Lopez Diez, J.: Low-Marangoni Low-Reynolds Numbers Capillary Flow Inside a Slender Liquid Bridge. Microgravity Sci. Technol., Vol. 3, pp. $222-230(1991)$.

29 Luengo, V., Meseguer; J., Parra, I.E.: An Experimental Study of the Stability of Long Axisymmetric Liquid Bridges between Solid Supports at Different Temperatures. Exp. Fluids, Vol. 34, pp. 412-417 (2003). 